\title{
Galactic Disk Structure and Metallicity from Mono-age Stellar Populations of LAMOST
}

\author{
Maosheng Xiang ${ }^{1,4}$, Xiaowei Liu ${ }^{2}$, Jianrong Shi ${ }^{1}$, Haibo Yuan $^{3}$, \\ Yang Huang ${ }^{2,4}$, Bingqiu Chen ${ }^{2}$ and Chun Wang ${ }^{2}$ \\ ${ }^{1}$ National Astronomical Observatories, Chinese Academy of Science msxiang@nao.cas.cn \\ ${ }^{2}$ Department of Astronomy, Peking University, China \\ ${ }^{3}$ Department of Astronomy, Beijing Normal University, China \\ ${ }^{4}$ LAMOST Fellow
}

\begin{abstract}
The LAMOST Galactic surveys provide robust stellar atmospheric parameters, abundances, masses and ages of millions of stars, allowing a unprecedented mapping of matter distribution, spatial structure, star formation rate, chemistry and kinematics of the Galaxy. In this proceeding we present structure and metallicity of the Galactic disk revealed by mono-age stellar populations within a few kilo-parsec of the solar neighborhood.
\end{abstract}

Keywords. Galaxy: general; Galaxy: disk; Galaxy: structure, Galaxy: abundances

\section{Introduction}

Characterizing the assemblage and evolution history of the Galactic disk is a fundamental task that becomes practicable in principle only until recently owing to the implementation of a few large-scale sky surveys, including photometric, spectroscopic and astrometric ones, that work together to deliver multi-dimensional parameters (3D positions, 3D motions, age, mass, metallicity and elemental abundances) of a huge, statistically meaningful number of stars. Among those parameters, stellar age is particularly a key to seek answers for many specific issues of the disk assemblage and evolution history, such as characteristic epochs of the disk formation, star formation and chemical enrichment histories, temporal evolution of disk morphology, etc. Nevertheless, delivering reliable stellar ages from the existing huge but usually inaccurate datasets are still challenging. As a consequence, robust and accurate age estimates for a large sample of stars were essentially absent in the past.

Due to fruitful efforts in deriving stellar atmospheric parameters, absolute magnitudes and abundances from low-resolution spectra of the LAMOST Galactic surveys, remarkable improvements have been achieved in both precision and accuracy of the stellar parameter estimates for millions of stars (Xiang et al. 2017a), which further leads to reliable age estimates of a million main sequence turn-off (MSTO) and subgiant stars of the Galactic disk. With this huge stellar sample, disk structure and stellar mass distribution, as well as metallicity and kinematics of mono-age stellar populations are explored. The results provide plenty of details and insights on the matter distribution, structure evolution, chemical and dynamical history of the Galactic disk. A combination of the LAMOST stellar parameters with parallax and proper motions from the coming data release (DR2+) of Gaia mission (Prusti et al. 2016) will provide better estimates of age, distance and tangential velocities for most LAMOST stars, thus is expected to promote significantly our knowledge of the assemblage and evolution history of our Galaxy. 

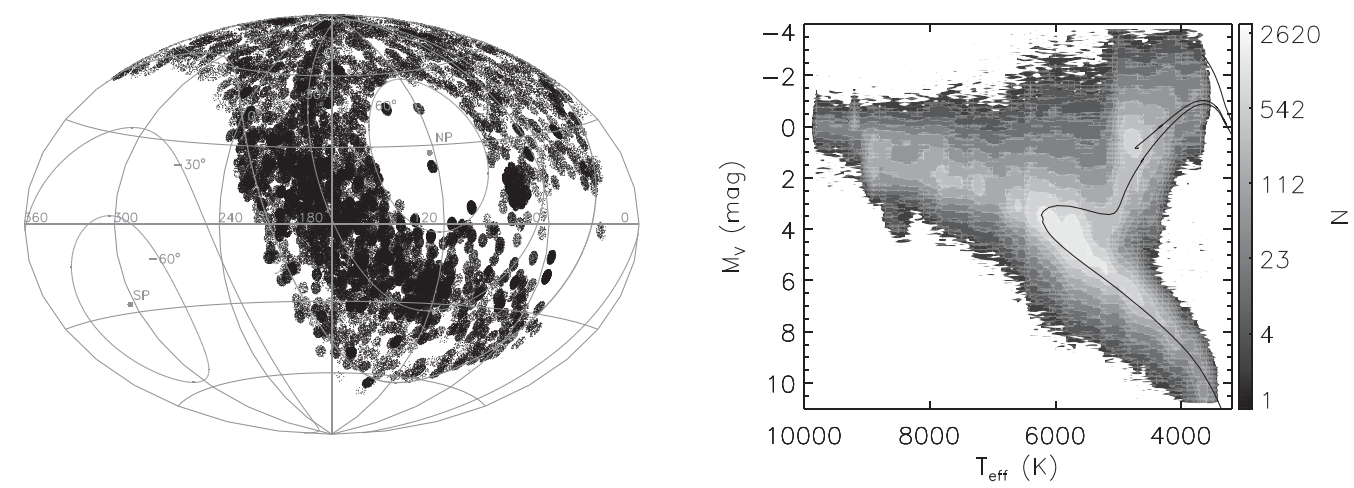

Figure 1. LAMOST sky coverage (left) in Galactic coordinate system and HR diagram (right) yielded by LSP3. Stellar isochrone of $4.5 \mathrm{Gyr}$ and solar metallicity from Rosenfield et al. (2016) is shown in the HR diagram.
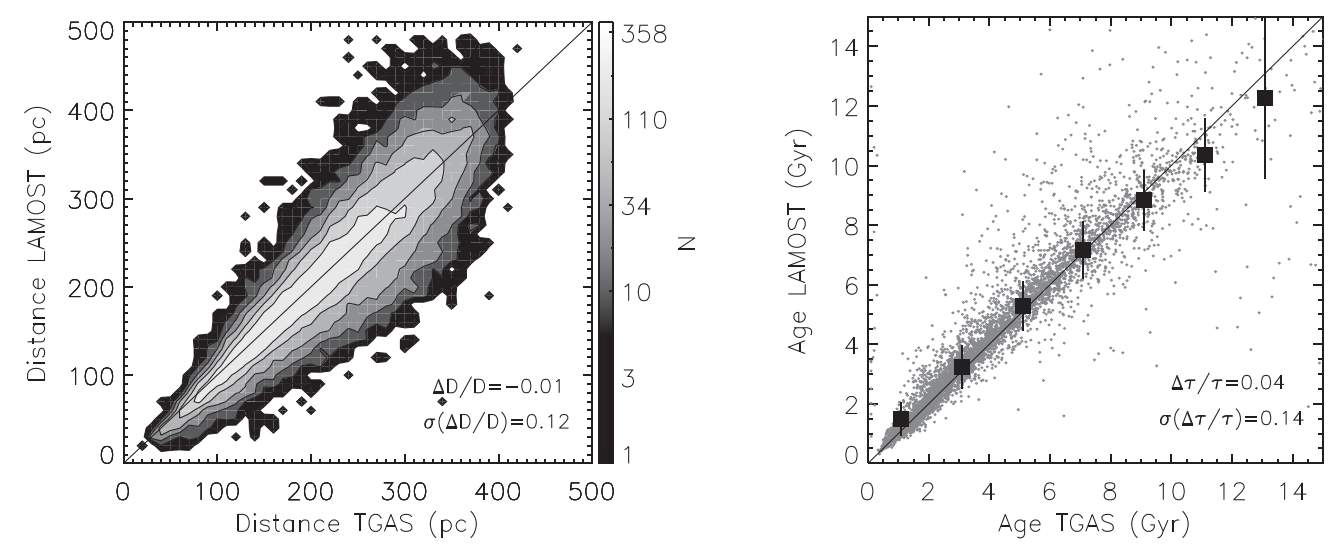

Figure 2. Comparisons of LAMOST stellar distance (left) and age (right) estimates with values derived based on TGAS parallax. Mean and dispersion of the relative differences are marked.

\section{The LAMOST data}

By June 2016, about 6.5 million low resolution $(R \sim 1800)$ stellar spectra for 4.4 million unique stars of $9-18.5 \mathrm{mag}$ in $r$-band are collected by LAMOST Galactic surveys (Zhao et al. 2012, Deng et al. 2012) with a spectral signal-to-noise ratio (SNR) higher than 10. The left panel of Fig. 1 shows the sky coverage. Stellar parameters, including radial velocity $V_{\mathrm{r}}$, effective temperature $T_{\text {eff }}$, surface gravity $(\log g)$, absolute magnitudes $\mathrm{M}_{V}, \mathrm{M}_{K_{\mathrm{s}}}$, metallicity $[\mathrm{Fe} / \mathrm{H}], \alpha$-element to iron abundance ratio $[\alpha / \mathrm{Fe}]$, carbon and nitrogen abundances $[\mathrm{C} / \mathrm{H}],[\mathrm{N} / \mathrm{H}]$, have been derived from the spectra with the LAMOST Stellar Parameter Pipeline developed at Peking University (LSP3; Xiang et al. 2015a, Li et al. 2016, Xiang et al. 2017a). Extensive examinations with high-resolution spectroscopy, asteroseismology, open clusters as well as duplicate observations, indicate that given a SNR higher than 50, LSP3 yields stellar parameters with uncertainties of only $5 \mathrm{~km} / \mathrm{s}, 100 \mathrm{~K}, 0.3 \mathrm{mag}, 0.15$ dex, 0.1 dex and 0.05 dex for $V_{\mathrm{r}}, T_{\text {eff }}, \mathrm{M}_{V}, \log g,[\mathrm{Fe} / \mathrm{H}]$ and $[\alpha / \mathrm{Fe}]$,respectively, and that LSP3 provides realistic parameter error estimates (Xiang et al. 2017b). The LSP3 stellar parameters, as well as interstellar extinction, distance and orbital parameters derived based on the LSP3 stellar parameters for stars targeted by the LAMOST Spectroscopic Survey of Galactic Anticentre (LSS-GAC; Liu et al. 2014, Yuan et al. 2015), a component of the LAMOST Galactic surveys, have been re- 

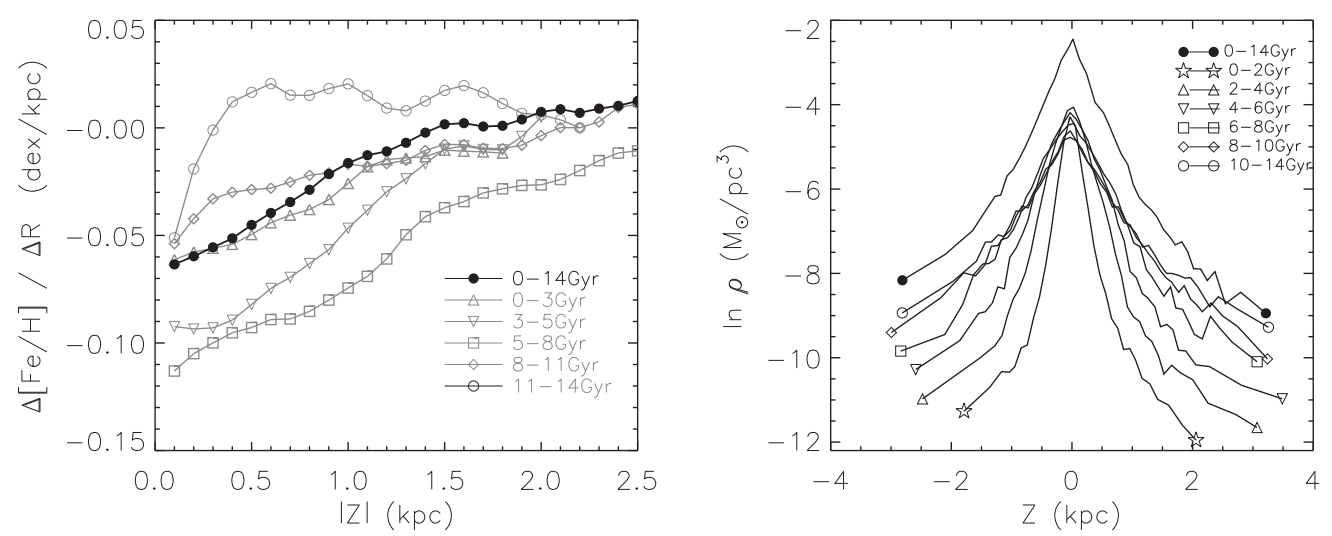

Figure 3. Left: radial metallicity gradient as a function of height away from the disk mid-plane for stellar populations of different ages. Right: vertical stellar mass density profile of different age populations in a Galactocentric radial annulus of $8.5<R<9.0 \mathrm{kpc}$.

leased as LSS-GAC value-added catalogues (Yuan et al. 2015, Xiang et al. 2017b), and can be downloaded via http://lamost973.pku.edu.cn/site/data. The right panel of Fig. 1 shows the distribution of stars in the $T_{\text {eff }}-\mathrm{M}_{V}$ diagram yielded by LSP3. The figure shows clear main sequence and red-giant branch, as well as a compact red clump. The diagram is in good agreement with stellar isochrones. The left panel of Fig. 2 shows a comparison of distance derived using the LSP3 $M_{\mathrm{V}}$ with those derived using Gaia TGAS parallax (Lindegren et al. 2016) for 50000 stars that have a TGAS-based absolute magnitude error smaller than $0.2 \mathrm{mag}$. The figure illustrates that systematic error of the LAMOST distance estimates using LSP3 $M_{\mathrm{V}}$ is negligible, and the random error is smaller than 12 per cent.

From the LSP3 stellar parameters, we have selected a sample of a million main sequence turn-off and subgiant (MSTO-SUB) stars of the disk $([\mathrm{Fe} / \mathrm{H}]>-1)$, which have a minimal SNR of 20, and a median SNR of about 60 . Stellar age and mass of the MSTO-SUB stars are estimated by matching stellar isochrones with a Bayesian algorithm using $T_{\text {eff }}$, $\mathrm{M}_{V},[\mathrm{Fe} / \mathrm{H}]$ and $[\alpha / \mathrm{Fe}]$. Extensive examinations indicate that half of the whole sample stars have an age uncertainty of only 20-30 per cent, and typical uncertainty of the mass estimates is a few per cent. The sample is going to be public available soon (Xiang et al. submitted). The right panel of Fig. 2 shows a comparison of stellar ages estimated using the LSP3 parameters with those using the TGAS parallax. The systematic difference is small, and the dispersion is 14 per cent only.

\section{Disk structure and metallicity from mono-age stellar populations}

The left panel of Fig. 3 shows the radial stellar metallicity gradients as a function of height above the disk mid-plane for populations of different ages. The gradients exhibit significant temporal evolution. The oldest stars have slightly positive (consistent with zero given an error of $0.01-0.02 \mathrm{dex} / \mathrm{kpc}$ ) gradients at almost all heights except the very thin disk region. Younger stars have negative gradients, which flatten with height. Stars of 5-8 Gyr exhibit the steepest gradients, while both younger and older stars have smaller gradients. This confirms the findings of Xiang et al. (2015b), who utilized a smaller sample with less accurate age estimates. The different behaviors of metallicity gradients between the oldest and the younger populations strongly suggest the existence of different phases 
in the assemblage history of the Galactic disk. The results provide important constrains on the disk formation and evolution scenarios.

A three-dimensional stellar mass density distribution of the disk within a few $\mathrm{kpc}$ of the solar neighborhood for each mono-age population has been mapped using the MSTO-SUB stars. In doing so, selection functions of the LAMOST Galactic surveys are corrected, and the initial mass function of Kroupa (2001) is used to transfer the mass of MSTO-SUB stars to the total stellar mass of the whole underlying stellar population. The right panel of Fig. 3 shows the vertical stellar mass density profiles of different age populations in the disk of $8.5<R<9.0 \mathrm{kpc}$. The figure shows a clear increasing trend of disk thickness with increasing stellar age. For almost all populations, the profiles can not be simply described by a single exponential function, which presents as a linear function in logarithm space, indicating that there are young thick disk and old thin disk, whose origins are particular interesting to further investigate.

\section{Acknowledgements}

This work is supported by National Key Basic Research Program of China 2014CB845700 and Joint Funds of the National Natural Science Foundation of China (Grant No. U1531244 and U1331120). Guoshoujing Telescope (the Large Sky Area Multi-Object Fiber Spectroscopic Telescope LAMOST) is a National Major Scientific Project built by the Chinese Academy of Sciences. Funding for the project has been provided by the National Development and Reform Commission. LAMOST is operated and managed by the National Astronomical Observatories, Chinese Academy of Sciences. The LAMOST FELLOWSHIP is supported by Special Funding for Advanced Users, budgeted and administrated by Center for Astronomical Mega-Science, Chinese Academy of Sciences (CAMS). This work has made use of data from the European Space Agency (ESA) mission Gaia (https://www.cosmos.esa.int/gaia), processed by the Gaia Data Processing and Analysis Consortium (DPAC, https://www.cosmos.esa.int/web/gaia/dpac/consortium). Funding for the DPAC has been provided by national institutions, in particular the institutions participating in the Gaia Multilateral Agreement.

\section{References}

Deng, L.-C., Newberg, H. J., Liu, C., et al., 2012, Research in Astronomy E Astrophysics, 12, 735

Gaia Collaboration, Prusti, T., de Bruijne, J. H. J., Brown, A. G. A., et al., 2016, A $6 A$, 595, 1

Haywood, M., Di Matteo, P., Lehnert, M. D., Katz, D., \& Gómez, A., 2013, A\&A, 560, 109

Li, J., Han, C., Xiang, M.-S., et al., 2016, Research in Astronomy \& Astrophysics, 16, 110

Lindegren, L., Lammers, U., Bastian, U., et al., 2016, A\&A, 595, 4

Liu, X.-W., Yuan, H.-B., Huo, Z.-Y., et al., 2014, in: S. Feltzing, G. Zhao, N. A. Walton \& P. A. Whitelock (eds.), Setting the scene for Gaia and LAMOST, Proc. IAU Symposium No. 298 , p. 310

Kroupa, P., 2001, MNRAS, 322, 231

Rosenfield, P., Marigo, P., Girardi, L., et al., 2016, ApJ, 822, 73

Xiang, M.-S., Liu, X.-W., Yuan, H.-B., et al., 2015, MNRAS, 448, 822

Xiang, M.-S., Liu, X.-W., Yuan, H.-B., et al., 2015, Research in Astronomy EG Astrophysics, 15, 1209

Xiang, M.-S., Liu, X.-W., Shi, J.-R., et al., 2017, MNRAS, 464, 3657

Xiang, M.-S., Liu, X.-W., Yuan, H.-B., et al., 2017, MNRAS, 467, 1890

Yuan, H.-B., Liu, X.-W., Huo, Z.-Y., et al., 2015, MNRAS, 448, 855

Zhao, G., Zhao, Y.-H., Chu, Y.-Q., Jing, Y.-P., \& Deng, L.-C., 2012, Research in Astronomy 6 Astrophysics, 12, 723 